\title{
The Elementary Particle Equation
}

\author{
Kennard Callender \\ Universidad Tecnológica de Panamá \\ Centro Regional de Veraguas
}

\begin{abstract}
An equation that describes the dynamics of elementary particles is formulated based on the hypothesis that physical space is a four-dimensional incompressible inviscid fluid.
\end{abstract}

\section{Contents}

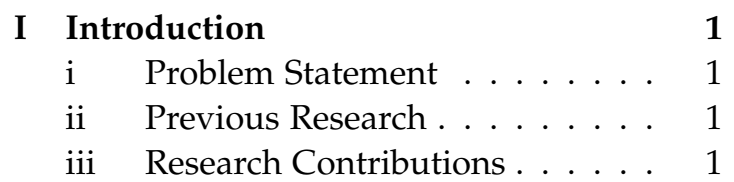

II The Elementary Particle Equation 2 i Postulates ......... 2

ii Quaternionic HJE . . . . . . . . 2

iii Derivation ......... 3

III Conclusion

\section{INTRODUCTION}

$\mathrm{N}$ EWTON's second law of motion was the first formulation of an equation that mathematically defined the dynamics of a point particle. It provided a deterministic explanation of the effect forces have on a body's motion. Nevertheless, the inability of this equation to accurately predict the motion of particles at the atomic level led to the formulation of Schrödinger's equation. Einstein, however, considered Schrödinger's equation to be an incomplete description of reality.

\section{i. Problem Statement}

In the present article, we formulate the Elementary Particle Equation. It is a realistic deterministic local equation that gives a complete description of elementary particle dynamics.
The equation is derived from the hypothesis that physical space is a four-dimensional incompressible inviscid fluid.

\section{ii. Previous Research}

The goal of our research program is to formulate a theory that explains and predicts all physical phenomena. In order to achieve this, we have proposed realistic deterministic local fundamental postulates that are based on the hypothesis that physical space is a fourdimensional incompressible inviscid fluid. Our program requires the theory to be derived from these postulates.

Recently, we formulated an interpretation of quantum mechanics [1] and an interpretation of the Lorentz transformation [2]. In those papers, we derived Schrödinger's equation and the Lorentz transformation from the postulates proposed by our research program. Those derivations suggest a realistic deterministic local equation, which would include Schrödinger's equation as a special case, can be formulated. Therefore, we proceed to do so in this article.

\section{iii. Research Contributions}

To the best of the author's knowledge, the following are original contributions from the present article:

- An equation, based on the hypothesis that physical space is a four-dimensional incompressible inviscid fluid, that gives a 
complete description of elementary particle dynamics.

- Formulating the quaternionic HamiltonJacobi equation and deriving the Elementary Particle Equation from it.

\section{The Elementary Particle EQUATION}

$\mathrm{I}$ $\mathrm{N}$ this section, we formulate the Elementary Particle Equation. First, we state the postulates proposed by our research program. Then, based on those postulates, we define the quaternionic Hamilton-Jacobi equation. Finally, we derive the Elementary Particle Equation from the quaternionic Hamilton-Jacobi equation.

\section{i. Postulates}

The postulates our research program proposes are the following:

- Time is absolute.

- Physical space is a four-dimensional incompressible inviscid fluid. Its flow is irrotational.

- Elementary particles always have a definite position, their movement is completely deterministic, and they are directly influenced only by their immediate surroundings.

- Elementary particles move at a constant speed along the streamlines of the fluid flow of physical space.

- Electromagnetic and gravitational waves propagate through physical space at a constant speed.

- Based on their perception at the macroscopic level, observers assume physical space is three-dimensional, and this assumption has unexpected effects on the measurements they perform.

These postulates were the ones used to derive Schrödinger's equation [1] and the Lorentz transformation [2]. The fact that those equations can be derived from these postulates gives us confidence they are a true representation of reality. ii. Quaternionic HJE

The quaternionic Hamilton-Jacobi equation (HJE) is formulated in this section. It is an extension of the complex conjugate HJE that was developed as part of the Four-Dimensional Fluid Space Interpretation of Quantum Mechanics [1].

The formulation presented here is for a single particle, moving in four-dimensional space, under the influence of a conservative force that depends only upon the spatial coordinates. For that case, we have that the quaternionic Hamilton-Jacobi equation is defined by

$$
H\left(\bar{q}, \frac{\partial S(\bar{q}, t)}{\partial \bar{q}}\right)+\frac{\partial S(\bar{q}, t)}{\partial t}=0
$$

where $H: \mathbb{H} \times \mathbb{H} \rightarrow \mathbb{H}$ is the quaternionic Hamiltonian function, $S: \mathbb{H} \times \mathbb{R} \rightarrow \mathbb{H}$ is Hamilton's quaternionic principal function, $\bar{q} \in \mathbb{H}$ is the generalized position in fourdimensional euclidean space, and $t \in \mathbb{R}$ is time. The conjugate momentum in four-dimensional euclidean space, $p \in \mathbb{H}$, corresponds to

$$
p=\frac{\partial S(\bar{q}, t)}{\partial \bar{q}}
$$

Because the quaternionic Hamiltonian does not depend explicitly on time, we have that Hamilton's quaternionic principal function can be written as

$$
S(\bar{q}, t)=W(\bar{q})-\frac{1}{2} E t
$$

where $W: \mathbb{H} \rightarrow \mathbb{H}$ is Hamilton's quaternionic characteristic function, and $E$ is a positive constant equal to the total energy of the system. From eqs. 2 and 3 we get that the conjugate momentum, $p$, is a function of $\bar{q}$ alone.

The quaternionic potential energy function, the total energy, and the initial position of the particle need to be specified to give a complete description of the system. Physically, the quaternionic HJE represents a particle of mass $m$ moving along the streamlines of an incompressible irrotational inviscid fluid flow in fourdimensional space. Therefore, the quaternionic $\mathrm{HJE}$ is an accurate mathematical formulation of the postulates from section 2.1. 


\section{iii. Derivation}

The Elementary Particle Equation will be derived in this section. All the variables and functions not defined here correspond to the ones defined in section 2.2. For the derivation, we need the conjugates of eqs. 1,2 , and 3 . Thus we have

$$
\begin{gathered}
\bar{H}\left(q, \frac{\partial \bar{S}(q, t)}{\partial q}\right)+\frac{\partial \bar{S}(q, t)}{\partial t}=0 \\
\bar{p}=\frac{\partial \bar{S}(q, t)}{\partial q} \\
\bar{S}(q, t)=\bar{W}(q)-\frac{1}{2} E t
\end{gathered}
$$

The total energy for a particle of mass $m$ under the influence of a conservative force can be written as

$$
E=\frac{p \bar{p}}{2 m}+V(\bar{q}, q)
$$

where $V: \mathbb{H} \times \mathbb{H} \rightarrow \mathbb{H}$ is the quaternionic potential energy function. The postulates in section 2.1 imply particles have a constant energy relative to physical space (because they move at a constant speed relative to it). It can be shown that, for a particle of mass $m$, this energy is equal to the total energy of the system, and it is given by

$$
E=m c^{2}
$$

The postulates from section 2.1 also indicate that particles must have a definite position at all times. Therefore, at $t=0$ we have

$$
\begin{aligned}
& q=q_{o} \\
& \bar{q}=\bar{q}_{o}
\end{aligned}
$$

The state function of the system is defined by

$$
\Psi(\bar{q}, q, t)=e^{i(S+\bar{S}) / \hbar}
$$

where $\Psi: \mathbb{H} \times \mathbb{H} \times \mathbb{R} \rightarrow \mathbb{H}, i$ is the versor of $q$ (or $\bar{q})$, and $\hbar$ is Planck's reduced constant. From eqs. 2, 3, 5, 6 and 11 we get

$$
i \hbar \frac{\partial \Psi}{\partial t}=E \Psi
$$

$$
\begin{gathered}
-i \hbar \frac{\partial \Psi}{\partial \bar{q}}=p \Psi \\
-i \hbar \frac{\partial \Psi}{\partial q}=\bar{p} \Psi \\
-\hbar^{2} \frac{\partial^{2} \Psi}{\partial q \partial \bar{q}}=p \bar{p} \Psi
\end{gathered}
$$

Multiplying eq. 7 by $\Psi$ gives

$$
E \Psi=\frac{p \bar{p}}{2 m} \Psi+V \Psi
$$

Finally, substituting eqs. 12 and 15 into eq. 16 gives us the Elementary Particle Equation:

$$
i \hbar \frac{\partial \Psi}{\partial t}=-\frac{\hbar^{2}}{2 m} \frac{\partial^{2} \Psi}{\partial q \partial \bar{q}}+V \Psi
$$

where the quaternionic potential energy function is required to be of the form

$$
V(\bar{q}, q)=\Omega(\bar{q}) \bar{\Omega}(q)=\frac{m c^{2}}{2}
$$

The general solution to the Elementary Particle Equation is given by

$$
\Psi=e^{i \sqrt{2 m}\left(\int \Omega d \bar{q}+\int \bar{\Omega} d q\right) / \hbar-i E t / \hbar}
$$

and applying eqs. 8, 9, and 10 (the initial conditions) gives

$$
\Psi=e^{i \sqrt{2 m}\left(\int_{\bar{q} \bar{q}_{0}}^{\bar{q}} \Omega d \bar{q}+\int_{q_{0}}^{q} \bar{\Omega} d q\right) / \hbar-i m c^{2} t / \hbar}
$$

As a final note, we have that Schrödinger's equation can be obtained from eq. 17 simply by setting the real part of $\bar{q}$ and $q$ equal to zero.

\section{Conclusion}

I $\mathrm{N}$ this article, we formulated the Elementary Particle Equation. It is an equation that gives a realistic, deterministic, local, and complete description of elementary particle dynamics. The equation is based on the hypothesis that physical space is a four-dimensional incompressible inviscid fluid. It was derived from the postulates of section 2.1 (via the quaternionic HJE). Those postulates were the 
same ones used to derive Schrödinger's equation [1] and the Lorentz transformation [2]. Therefore, we have confidence they are correct and that the Elementary Particle Equation gives an accurate description of the physical world.

\section{DedicATION}

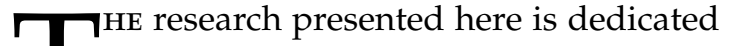
to the memory of my father, Dr. Lorenzo

1 León Callender López, who always supported me and was there for me. Without him, this research would not have been possible.

\section{REFERENCES}

[1] Callender, Kennard. The Four-Dimensional Fluid Space Interpretation of Quantum Mechanics. OSF Preprints. September 10, 2020. doi:10.31219/osf.io/2y3t5.

[2] Callender, Kennard. Why is the Speed of Light in Vacuum a Universal Physical Constant? OSF Preprints. September 18, 2020. doi:10.31219/osf.io/6rhcb. 